\title{
Stochastic generation of particle structures with controlled degree of heterogeneity
}

\section{Journal Article}

Author(s):

Schenker, Iwan; Filser, Frank T.; Gauckler, Ludwig J.

Publication date:

2010

Permanent link:

https://doi.org/10.3929/ethz-b-000018650

Rights / license:

In Copyright - Non-Commercial Use Permitted

Originally published in:

Granular Matter 12(4), https://doi.org/10.1007/s10035-010-0188-5 


\title{
Stochastic generation of particle structures with controlled degree of heterogeneity
}

\author{
Iwan Schenker • Frank T. Filser • Ludwig J. Gauckler
}

Received: 11 September 2009 / Published online: 17 April 2010

(C) Springer-Verlag 2010

\begin{abstract}
The recently developed void expansion method (VEM) allows for an efficient generation of porous packings of spherical particles over a wide range of volume fractions. The method is based on a random placement of the structural particles under addition of much smaller "void-particles" whose radii are repeatedly increased during the void expansion. Thereby, they rearrange the structural particles until formation of a dense particle packing and introduce local heterogeneities in the structure. In this paper, microstructures with volume fractions between 0.4 and 0.6 produced by VEM are analyzed with respect to their degree of heterogeneity (DOH). In particular, the influence of the void- to structural particle number ratio, which constitutes a principal VEM-parameter, on the DOH is studied. The DOH is quantified using the pore size distribution, the Voronoi volume distribution and the density-fluctuation method in conjunction with fit functions or integral measures. This analysis has revealed that for volume fractions between 0.4 and 0.55 the void-particle number allows for a quasi-continuous adjustment of the DOH. Additionally, the DOH-range of VEM-generated microstructures with a volume fraction of 0.4 is compared to the range covered by microstructures generated using previous Brownian dynamics simulations, which represent the structure of coagulated colloidal suspensions. Both sets of microstructures cover similarly broad and overlapping DOH-ranges, which allows concluding that VEM is an efficient method to stochastically reproduce colloidal microstructures with varying $\mathrm{DOH}$.
\end{abstract}

I. Schenker $(\bowtie) \cdot$ F. T. Filser · L. J. Gauckler

Nonmetallic Materials, Department of Materials, ETH Zurich, 8093, Zurich, Switzerland

e-mail: iwan.schenker@mat.ethz.ch
Keywords Degree of heterogeneity - Discrete element method · Microstructure generation · Porosity · Void expansion method

\section{Introduction}

The mechanical properties of colloidal particle gels are of great importance in nature and in many of today's technologies. Sediments [1], clay [2], some food [3] or paints and coatings [4] are but a few examples. Typically, colloidal gels exhibit very complex mechanical behaviors such as shear thickening [5], thixotropy [6] or aging [7]. From a scientific perspective, these mechanical properties are often studied using random sphere packings. They provide ubiquitous model systems for colloids-or granular materials in general-and allow for a systematic study of the mechanical properties as a function of the various parameters as, for example, the volume fraction [8], the particle size distribution [9], material properties such as the particles' friction coefficients [10] or adhesive forces [11].

Our present research focuses on the influence of the microstructural arrangement of the primary particles on the structure's mechanical properties. This influence is often observed implicitly in experimental and simulated mechanical tests on gels or particle packings differing in preparation history. Macroscopic stress profiles, for example, were found to depend strongly on the sample preparation procedure and thus on the microstructure [12]. Franks et al. [13] have investigated the mechanical properties of sedimented aggregates of submicron alumina particles and, in particular, the influence of the aggregate size (i.e. the microstructure) on the rheological properties. They found that sediments formed from larger aggregates exhibit higher shear and compressive yield 
strengths. In [14-16], the influence of the heterogeneity of a coagulated colloidal suspension on its mechanical properties was investigated. It was shown that more heterogeneous microstructures exhibit up to ten times higher elastic properties and yield strengths than their more homogeneous counterparts at equal volume fraction. These experiments have systematically shown that the local arrangement of the powder particles has a strong influence on the mechanical properties. The relation between the microstructure and the mechanical properties, however, is still an open question.

A precondition for any methodical study of the relation between the microstructure of any granular material and its mechanical properties is the possibility to generate particle packings with a controlled degree of heterogeneity (DOH). For colloidal particle structures, this reproducible control of the heterogeneity is experimentally achieved using an enzyme catalyzed gelation method (DCC $=$ direct coagulation casting $[17,18])$. DCC allows for an undisturbed coagulation of electrostatically stabilized colloidal suspensions to stiff microstructures by an in situ transition of the interparticle potential from repulsive to attractive. This can be done along two principal pathways: shifting the $\mathrm{pH}$ of the suspension to the particles' isoelectric point ( $\Delta \mathrm{pH}$-method) or increasing the ionic strength in the suspension at constant $\mathrm{pH}(\Delta \mathrm{I}$-method), which compresses the Debye length of the repulsive inter-particle potential. The first method leads to more homogeneous microstructures through diffusionlimited aggregation. The second method produces more heterogeneous microstructures via reaction-rate-limited aggregation [15].

A variation of the $\Delta \mathrm{pH}$-method producing microstructures with higher DOH consists in admixing alkali-swellable polymer (ASP) particles to the powder particles under acidic conditions [19]. The initial diameter of the ASP particles is small (approximately $80 \mathrm{~nm}$ ) in comparison to the diameter of the structural particles $(400 \mathrm{~nm})$. The ASP particles swell upon increasing $\mathrm{pH}$ during the internal gelling reaction and unfold to roughly $700 \mathrm{~nm}$ in diameter, thereby pushing the structural particles in their vicinity, creating larger pores and thus producing more heterogeneous microstructures.

One way to investigate the microstructure-dependent mechanical properties of coagulated colloidal structures is by computational means, such as the discrete element method or molecular dynamics. These methods take intrinsically account of the colloid's particulate nature but require to be provided with the initial particle configurations. In the case of gravitationally stable random particle packings with volume fractions between random loose $\left(\Phi_{R L P} \approx 0.55\right.$ [20]) and random close packing $\left(\Phi_{R C P} \approx 0.64\right.$ [21]) a large variety of algorithms exists. In [22], for example, an overview of available approaches is given. Basically, they can be categorized into two main branches: dynamic techniques and constructive algorithms. Dynamic techniques, to which the method used in this study belongs, rely on a gradual swelling of the particles or shrinking of the simulation box. Constructive methods, such as the method introduced in [22], construct densely packed particle assemblies using geometrical calculations. Volume fractions below $\Phi_{R L P}$ were achieved by molecular dynamics simulations using long-range attractive interactions between the particles and high friction coefficients [23].

Despite the large amount of structure generation methods, to our knowledge, none of them has been shown to provide the possibility to control the microstructural arrangement of the particles independently of other parameters such as the volume fraction or the particle size distribution. This control, however, is crucial to our study of the microstructure-dependent mechanical properties and was a motivation to develop the void expansion method (VEM) presented in [24]. The method was originally inspired by the generation of heterogeneous microstructures using ASP particles and was shown to allow for a fast and efficient computational generation of porous particle structures over a broad range of volume fractions.

In this paper, particle packings with volume fractions between 0.4 and 0.6 generated using the VEM are further analyzed in terms of their $\mathrm{DOH}$. The $\mathrm{DOH}$, introduced in [25], represents an abstract concept quantitatively describing the heterogeneity of a particle arrangement by scalar measures. In order to quantify the $\mathrm{DOH}$, three structure characterization methods in combination with parameters in fit functions or integral measures are used: the pore size distribution, the Voronoi volume distribution and the density-fluctuation method. For volume fractions between 0.4 and 0.55 , we show that the number of void-particles strongly influences the $\mathrm{DOH}$ of the final microstructure. Additionally, for a volume fraction of 0.4 , the degrees of heterogeneity of structures generated using VEM and previous Brownian dynamics (BD) simulations [26,27] are compared. The BD microstructures represent coagulated colloidal microstructures, for which the DOH was shown to depend on the presence and depth of a secondary minimum in the inter-particle potential described by the Derjaguin-Landau-Verweg-Overbeek (DLVO) theory [28]. In contrast to these BD-simulations that simulate the coagulation using widely accepted physical laws and theories, VEM is a stochastic method that has the advantage of its computational efficiency. We found that the $\mathrm{DOH}$-ranges of the VEM- and the BD-microstructures overlap to a large extent with the range of the VEM-microstructures shifted to slightly higher values. This indicates that VEM is well-suited to reproduce coagulated colloidal microstructures with varying $\mathrm{DOH}$. 


\section{Materials and methods}

\subsection{Void expansion method}

The void expansion method is implemented using the particle flow code in three dimensions $\left(\mathrm{PFC}^{3 \mathrm{D}}\right)$ from Itasca Consulting Group, Inc., Minneapolis, Minnesota, USA [29]. PFC ${ }^{3 \mathrm{D}}$ is based on the discrete element method [30], which allows a modelling of the movement of assemblies of rigid spherical particles. In $\mathrm{PFC}^{3 \mathrm{D}}$, a central-difference scheme is used to numerically integrate the accelerations and velocities of the particles and thus to determine their dynamic behavior. The forces on the particles included in our model arise from a linear elastic contact law between the particles and damping. The contact law is characterized by the particles' normal and shear stiffness $k_{n}$ and $k_{s}$, respectively and the damping force is adjusted via the damping coefficient $d$ [31].

VEM relies on two kinds of particles: "structural particles" and "void-particles". The structural particles, with normal stiffness $k_{n, S}$ and shear stiffness $k_{s, S}$, constitute the final microstructures, whereas the void-particles (normal and shear stiffness $k_{n, V}$ and $k_{s, V}$, respectively) are only used during structure generation. The edge length $L_{b o x}$ of the cubic simulation box with periodic boundary conditions is calculated using the number of structural particles $N_{S}$, their radius $r_{S}$ and their volume fraction $\Phi_{S}$ via

$L_{\text {box }}=r_{s}\left(\frac{4 N_{S} \pi}{3 \Phi_{S}}\right)^{1 / 3}$

The $N_{S}$ structural particles are randomly placed in the simulation box. In $\mathrm{PFC}^{3 \mathrm{D}}$, particles are not allowed to overlap during their generation. Thus, in order to achieve volume fractions higher than approximately 0.35 , the structural particles are generated with a reduced initial radius of $r_{S} /(m+1), m=10$ in our simulations. The $N_{V}$ void-particles having a radius $r_{V} \ll r_{S}$ are randomly added to the structural particles. After generation of all the particles, the radius of the structural particles is increased by means of $m$ repeated radius blow-up steps. At each step, the initial particle radius is added to the current radius, followed by an equilibration of the structure until, after $m$ steps, the final particle radius $r_{S}$ is reached.

After the structural particles have reached their final size, the radius of the void-particles is cyclically increased by adding their initial radius $r_{V}$ to their current radius in alternation with the performance of 20,000 calculation steps in order to allow for a relaxation of the structure. This cyclic increase of the void-particle radius simulates the swelling of the ASP particles in the experiment and causes the structural particles to rearrange and to get in contact with each other.

The iteration is generally done until the structural and void-particles are densely packed and any further increase in the void-particle radius leads to a compaction of the struc- tural particles. This is reflected by an increasing internal strain energy, which essentially depends on the stiffness of the void- and the structural particles. In this paper, VEM-microstructures having a specific average coordination number are chosen for further analysis. For a volume fraction of 0.4 , an average coordination number of $C N_{0.4}=4.7$ is targeted. This value corresponds to the average coordination number of the microstructures resulting from the aforementioned BDsimulations, thus allowing for a direct comparison of their degrees of heterogeneity. For volume fractions above 0.4, the according coordination numbers are determined using a linear interpolation between $C N_{0.4}$ and $C N_{R C P}=6$ [32]. All analyses are performed on the structural particles alone, after deletion of the void-particles. As shown in [24], the evolution of $C N$ as a function of the void-particle radius depends on the void-particle number $N_{V}$. The range of the $N_{V}$-values that allow reaching the targeted $C N$ is discussed in Sect. 3.2 in terms of particle overlaps.

The simulation parameters are summarized in Table 1. In order to reduce the inertia of the void-particles their density $\rho_{V}$ was set ten times smaller than the density of the structural particles $\rho_{S}$, for which the density of bulk alumina was chosen. The inter-particle friction coefficient $\mu$ was set to zero in order not to impede any particle rearrangements.

\subsection{Quantification of the DOH}

Three distinct structural characterization methods are used to analyze and assess the heterogeneity of the VEMmicrostructures [25]: the pore size distribution, the densityfluctuation method and the distribution of Voronoi volumes. Each method, in conjunction with fit functions or integral measures, provides a scalar measure that captures and quantifies the $\mathrm{DOH}$ of a microstructure.

\subsubsection{Pore size distribution}

The pore size distribution is calculated using the exclusion probability $E_{V}(r)$ [33]. It is defined as the probability of inserting a "test" particle of radius $r$ at some arbitrary position in the pore space of a microstructure and is estimated using a Monte Carlo approach. The probability $P\left(r_{P}>r\right)$ of finding a pore with radius $r_{P}$ larger than $r$ is obtained using $P\left(r_{P}>r\right)=\sum_{r^{\prime}>r_{P}} E_{V}\left(r^{\prime}\right)$. For coagulated colloidal suspensions resulting from BD-simulations it was shown that $P\left(r_{P}>r\right)$ follows a complementary error function given by

$P\left(r_{P}>r\right)=1-\operatorname{erf}\left(\frac{r / r_{0}-b}{a \sqrt{2}}\right)$,

with $a$ and $b$ the standard deviation and the mean value, respectively. Parameter $a$ was shown to nicely reflect the structure's DOH, where increasing values of $a$ indicate an increasing DOH. 
Table 1 Simulation parameters

\begin{tabular}{lll}
\hline Parameter & Symbol & Value \\
\hline Number of particles & $N_{S}$ & 8,000 \\
Particle radius & $r_{S}$ & $2.5 \times 10^{-7} \mathrm{~m}$ \\
Normal structural particle stiffness & $k_{n, S}$ & $10^{3} \mathrm{~N} / \mathrm{m}$ \\
Shear structural particle stiffness & $k_{s, S}$ & $10^{-2} \mathrm{~N} / \mathrm{m}$ \\
Number of void-particles & $N_{V}$ & $1,000-16,000$ \\
Normal void-particle stiffness & $k_{n, V}$ & $10^{2} \mathrm{~N} / \mathrm{m}$ \\
Shear void-particle stiffness & $k_{s, V}$ & $10^{-2} \mathrm{~N} / \mathrm{m}$ \\
Damping coefficient & $d$ & 0.7 \\
Friction coefficient & $\mu$ & 0.0 \\
Volume fraction & $\Phi_{S}$ & $0.4-0.6$ \\
Structural particle density & $\rho_{S}$ & $3,690 \mathrm{~kg} / \mathrm{m}^{3}$ \\
Void-particle density & $\rho_{V}$ & $369 \mathrm{~kg} / \mathrm{m}^{3}$ \\
\hline
\end{tabular}

\subsubsection{Density fluctuation method}

The density-fluctuation method statistically analyzes the spatial distribution of the particle centers as a function of grid spacing. Therefor, the cubic simulation box is subdivided into $n_{c}^{3}$ cells where $n_{c}=2, \ldots, n_{c}^{\max }\left(n_{c}^{\max }=33\right.$, cf. [25]). The standard deviation $\sigma_{p p c}$ normalized by the average particle number $E_{p p c}$ as a function of grid spacing was shown to depend on the $\mathrm{DOH}$ of the microstructure and the integral $I_{d f}$ over these curves (Eq. (3)) provides a measure of the $\mathrm{DOH}$.

$I_{d f}=\sum_{n_{c} \leq n_{c}^{\max }} \frac{\sigma_{p p c}\left(n_{c}\right)}{E_{p p c}}$

\subsubsection{Voronoi volume distribution}

The Voronoi volume of a particle is the volume given by the union of all points in pore space closer to the surface of this particle than to any other particle [34]. The Voronoi tessellation thus divides a set of particles into a set of space-filling, non-overlapping and convex polyhedrons. In this study, the polyhedron volumes are determined using the Qhull package [35]. Following a statistical mechanics approach, Aste and Di Matteo have shown in [36] that the Voronoi volume distribution of a particle set follows a so-called $k$-gamma distribution given by

$f\left(V^{f}, k\right)=\frac{k^{k}}{\Gamma(k)} \frac{\left(V^{f}\right)^{k-1}}{\left(\bar{V}^{f}\right)^{k}} \exp \left(-k \frac{V^{f}}{\bar{V}^{f}}\right)$.

The Voronoi free volume $V^{f}=V-V_{\min }$ is the difference between a particle's Voronoi volume $V$ and the minimum volume of a Voronoi cell $V_{\min }$, which is achieved for a regular close packing and is given by $V_{\min }=1.325 V_{\text {sphere }}$, with
$V_{\text {sphere }}$ the volume of a particle. The mean Voronoi free volume $\bar{V}^{f}$ is a scaling parameter and the free parameter $k$ characterizes the shape of the curve that very sensitively depends on the structural organization of the particles. In [25], the $k$-gamma distribution was used to fit the Voronoi volume distribution of BD-microstructures. A very good agreement between data and fit was obtained and it was shown that parameter $k$ reflects very sensitively the $\mathrm{DOH}$ of the various microstructures.

\section{Results and discussion}

A qualitative impression of the influence of the void-particle number $N_{V}$ on the resulting microstructures is given in Fig. 1, presenting slices with a thickness of three particle layers and equal volume fraction of 0.4 . In the upper row, slices through VEM-microstructures generated using $N_{V}=16,000$ (left) and $N_{V}=1,000$ (right), respectively, are shown. In the left slice, the particles are rather uniformly distributed, whereas the particles in the slice on the right are locally more densely packed and thus larger voids can be observed. Very similar properties are observed for the BD-microstructures inserted in the lower row. On the left, the most homogeneous (surface potential $\Psi_{0}=0 \mathrm{mV}$ ) and, on the right, the most heterogeneous BD-microstructure $\left(\Psi_{0}=15 \mathrm{mV}\right)$ are shown. Figure 1 suggests that the use of larger void-particle numbers leads to more homogeneous microstructures and that VEM allows obtaining very similar microstructures as BD in terms of heterogeneity, as supported by the quantitative analyses in Sect. 3.1. In Sect. 3.2, the DOH of the microstructures generated using VEM and BD are compared and, in Sect. 3.3, the possibility to generate microstructures with a varying DOH for volume fraction above 0.4 is investigated. 

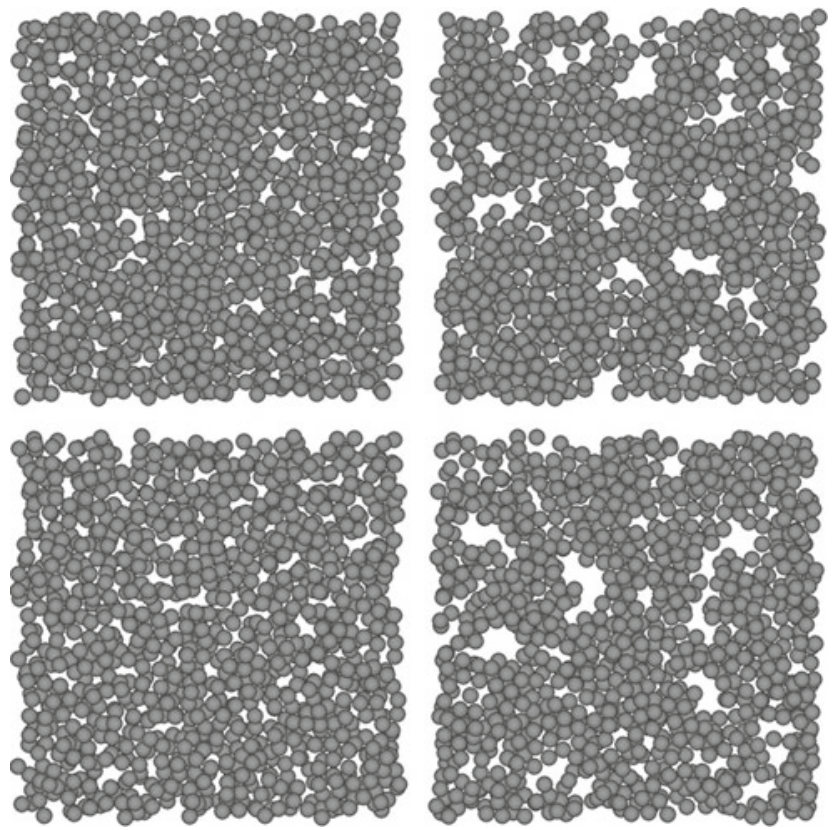

Fig. 1 Slices through VEM-microstructures (upper row) with a more homogeneous microstructure $\left(N_{V}=16,000\right.$, left $)$ and a more heterogeneous microstructure $\left(N_{V}=1,000\right.$, right $)$. The lower row shows the most homogeneous $\left(\Psi_{0}=0 \mathrm{mV}\right.$, left $)$ and most heterogeneous microstructure $\left(\Psi_{0}=15 \mathrm{mV}\right.$, right $)$ resulting from earlier BD-simulations [26]. Slice thickness: three particle diameters; particle diameter $=0.5 \mu \mathrm{m}$, volume fraction $=0.4$ (for all structures)

\subsection{Influence of the void-particle number}

In this section, the results obtained for the three distinct methods used to quantify the DOH are presented in detail for the VEM-microstructures with a volume fraction of 0.4 and varying void-particle number $N_{V}$.

\subsubsection{Pore size distribution}

The probability $P\left(r_{P}>r\right)$ of finding pores with a radius $r_{P}$ larger than $r$ is shown in Fig. 2 for various VEMmicrostructures with void-particle numbers ranging from 1,000 to 16,000 (symbols). The data is shown as a function of $r$ normalized by the particle radius $r_{S}$. For a given pore radius $r_{P}>0, P\left(r_{P}>r\right)$ decreases for increasing void-particle numbers $N_{V}$. The probability of finding pores with a radius larger than $0.5 r_{S}$ is 1.6 times higher in the $N_{V}=1,000$ than in the $N_{V}=16,000$ microstructure. Finding pores larger than $0.75 r_{S}$ and $1.0 r_{S}$ is 4.3 and 18.7 times more probable in the microstructure with $N_{V}=1,000$ than in the one with $N_{V}=16,000$, respectively. The solid lines in Fig. 2 represent the complementary error function fit curves obtained using Eq. (2). Fit parameters $a$ and $b$ and the corresponding $R_{a, b}^{2}$-values are summarized in Table 2. The $R_{a, b}^{2}$-values close to one indicate

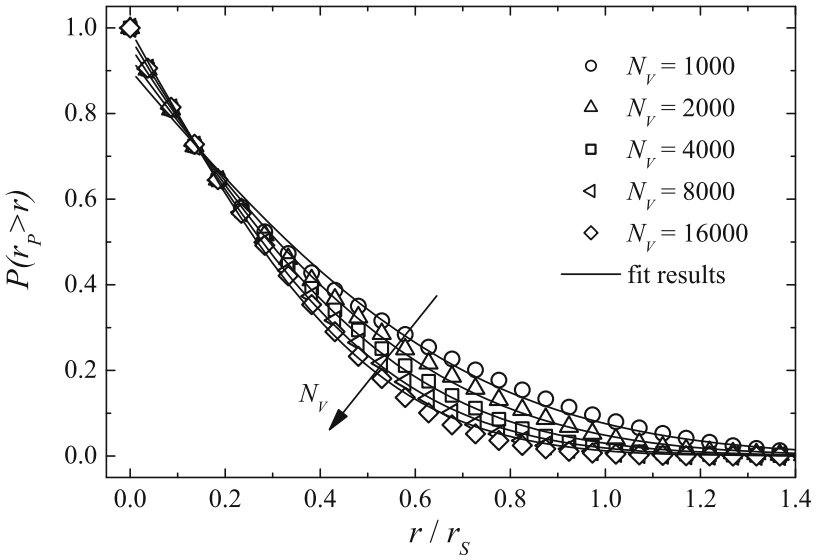

Fig. 2 Probability $P\left(r_{P}>r\right)$ of finding pores with a radius $r_{P}$ larger than $r$ versus $r / r_{S}$ obtained using the pore size distribution for the various VEM-microstructures with $\Phi_{S}=0.4$ (symbols). Solid lines denote the corresponding fits using a complementary error function (Eq. 2)

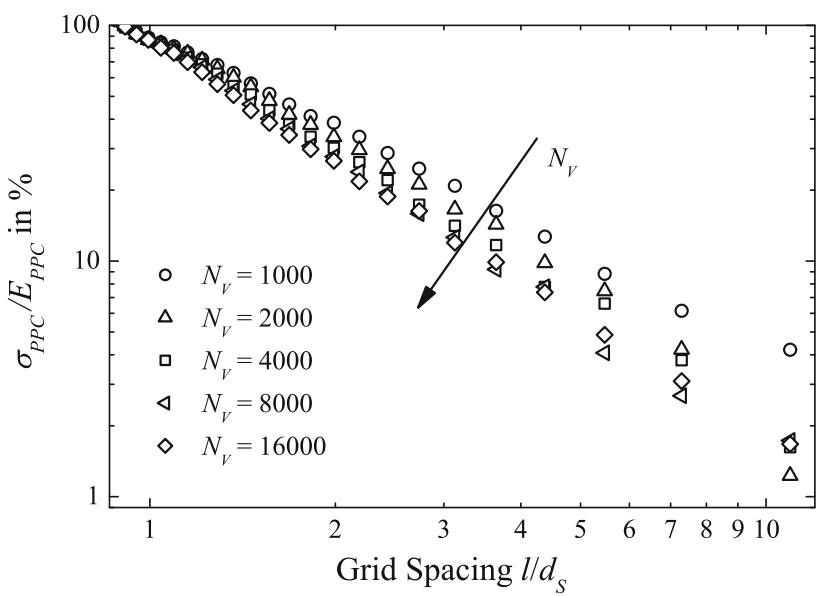

Fig. 3 Density fluctuations as a function of grid spacing for the various VEM-microstructures with $\Phi_{S}=0.4$

excellent fits. The largest value $a$ is found for the microstructure generated using 1,000 void-particles. For increasing values of $N_{V}$, parameter $a$ decreases, which reflects a decreasing probability of finding larger pores and thus a decreasing $\mathrm{DOH}$.

\subsubsection{Density fluctuation method}

The density fluctuations of the various VEM-microstructures are presented in Fig. 3 as a function of the grid spacing normalized by the particle diameter $d_{S}=2 r_{S}$. The density fluctuations decrease with increasing void-particle number. This behavior is quantified using $I_{d f}$ as given in Eq. (3). The values are summarized in Table $2 . I_{d f}$ increases with decreas- 
Table 2 Measures of the DOH as a function of the void-particle number $N_{V}$ : fit parameters $a$ and $k$, corresponding to the widths of the pore size and Voronoi volume distribution, respectively, and the integral over the density fluctuation curves $I_{d f}$

\begin{tabular}{|c|c|c|c|c|c|c|}
\hline$N_{V}$ & $a$ & $b\left(10^{-2}\right)$ & $R_{a, b}^{2}$ & $I_{d f}$ & $k$ & $R_{k}^{2}$ \\
\hline 1,000 & 0.605 & -7.44 & 0.997 & 24.2 & 2.46 & 0.977 \\
\hline 2,000 & 0.530 & -4.63 & 0.999 & 23.6 & 2.99 & 0.995 \\
\hline 4,000 & 0.472 & -2.56 & 0.999 & 23.1 & 3.61 & 0.998 \\
\hline 8,000 & 0.431 & -1.21 & 0.999 & 22.7 & 4.67 & 0.999 \\
\hline 11,000 & 0.416 & -0.652 & 0.999 & 22.6 & 5.02 & 0.999 \\
\hline 13,000 & 0.411 & -0.458 & 0.998 & 22.6 & 5.50 & 0.999 \\
\hline 16,000 & 0.401 & -0.0227 & 0.999 & 22.5 & 5.74 & 0.998 \\
\hline
\end{tabular}

Additionally, the second fit parameter of the cumulative pore size distribution $(b)$ and the corresponding $R^{2}$-values are shown

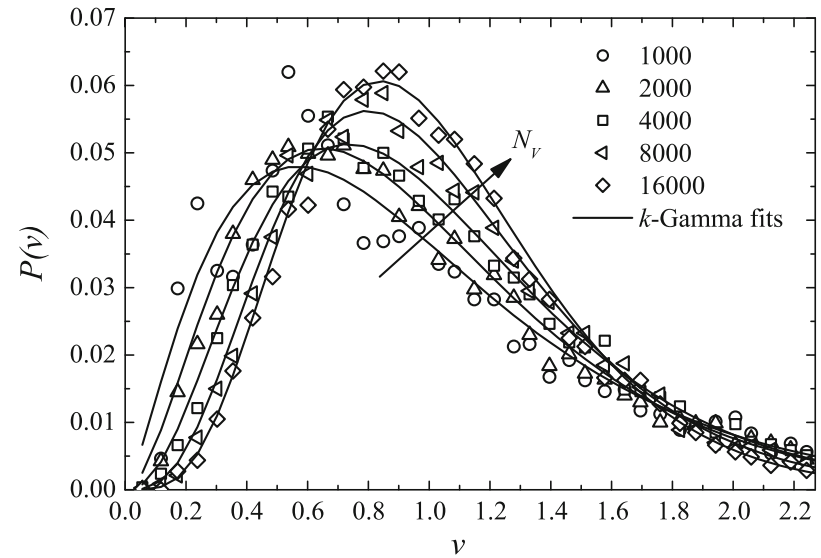

Fig. 4 Voronoi volume distribution $P(v)($ symbols $)$ as a function of $v$ and corresponding $k$-gamma fits (lines) for various VEM-microstructures $\left(\Phi_{S}=0.4\right)$

ing $N_{V}$ and thereby reflects an increasingly heterogeneous repartition of the particles.

\subsubsection{Voronoi volume distribution}

The Voronoi volume distribution $P(v)$ of the various VEMmicrostructures is shown in Fig. 4 for void-particle numbers ranging between 1,000 and 16,000 (symbols) as a function of $v=\frac{V^{f}}{\bar{V} f}$, the free Voronoi volume normalized by the mean free volume. For increasing $N_{V}$, the peak height increases and, consequently, the width of the curve decreases. This behavior indicates that more homogeneous microstructures are found toward increasing $N_{V}$, which is confirmed by a fit of the curves using the $k$-gamma distribution given in Eq. (4). The fits are shown as lines in Fig. 4. The corresponding values for the parameter $k$, summarized in Table 2, increase for increasing $N_{V}$ reflecting the shift toward more homogeneous microstructures. Very good fit results are achieved as indicated by the $R_{k}^{2}$-values.

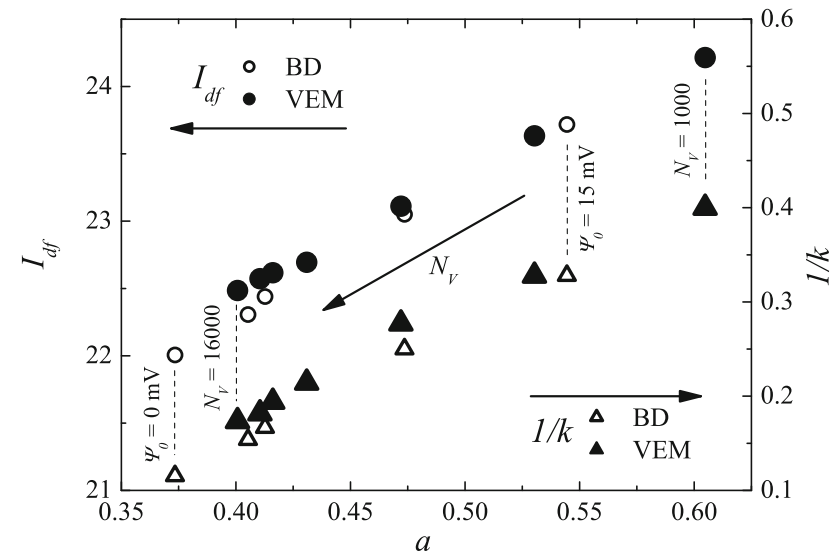

Fig. 5 Interdependence of the DOH-measures $\left(\Phi_{S}=0.4\right)$ : Integral over the density fluctuation curves $I_{d f}$ (circles, left scale) and width of the Voronoi volume distribution $1 / k$ (triangles, right scale) as a function of the width of the pore size distribution $a$ for the various VEM-microstructures (full symbols) and for the BD-microstructures (open symbols [25]) at $\Phi_{S}=0.4$

\subsection{DOH for VEM- and BD-structures at volume fraction 0.4}

The interdependence between the three measures of the DOH is shown in Fig. 5. On the left scale, the integral over the density fluctuation curves $I_{d f}$ is shown as a function of the width of the pore size distribution, given by parameter $a$. The corresponding values are shown as circles. The right scale presents $1 / k$ reflecting the width of the Voronoi volume distribution in dependence of $a$, shown as triangles. Full symbols denote the values obtained for the VEM-microstructures.

The linear arrangement of the two curves suggests a pairwise affine relation between the various DOH. Furthermore, the values found for the VEM-microstructures are very close to those obtained for the BD-microstructures, which are inserted as open symbols. These two points substantiate the equivalence of the three methods as already observed in [25].

The DOH-range covered by the VEM-microstructures, in terms of parameter $a$, extends from 0.4 to 0.6 . This overlaps to 


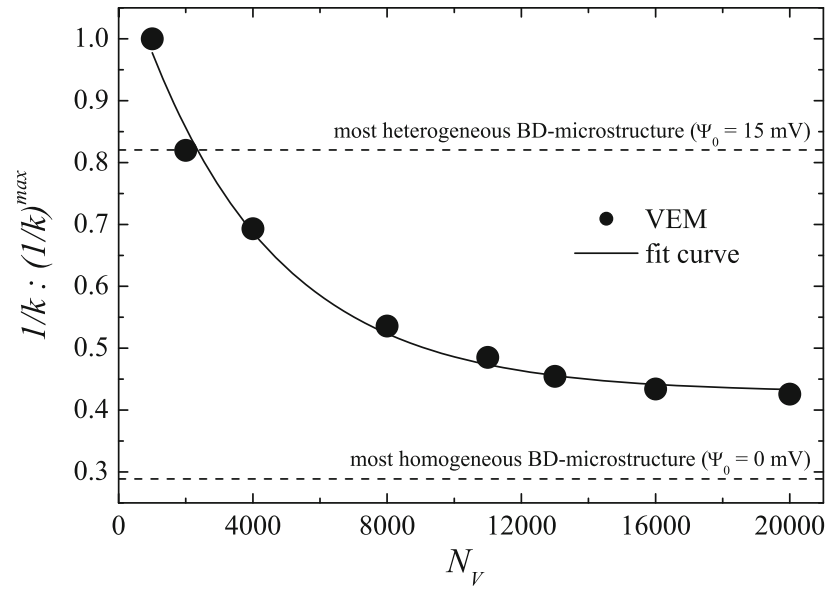

Fig. 6 DOH-measure $1 / k$ relative to $(1 / k)^{\max }$ as a function of the void-particle number $N_{V}$ for $\Phi_{S}=0.4$ (symbols). The dashed lines denote the corresponding values of the most and least heterogeneous BD-microstructure [25]

a large extent with the range of the BD-microstructures from 0.37 to 0.54 . Thus, the range covered by VEM is roughly $20 \%$ broader starting at a slightly higher $\mathrm{DOH}$ than the range of the BD-microstructures considered in this work.

The sensitivity of the DOH on $N_{V}$ is demonstrated in Fig. 6 presenting $1 / k$ relative to its maximum value $(1 / k)^{\max }$, achieved for $N_{V}=1,000$, as a function of $N_{V}$. After a strong initial decrease for small values of $N_{V}, 1 / k$ levels off toward higher values of $N_{V}$. A further increase of $N_{V}$ has only a negligible influence on the $\mathrm{DOH}$ as shown by the insertion of the additional $1 / k$-value for $N_{V}=20,000$. Indeed, an increase in $N_{V}$ from 16,000 to 20,000 decreases the DOH by only $2 \%$, which suggests that the $\mathrm{DOH}$ of the most homogeneous $\mathrm{BD}$-microstructure, represented by the lower dashed line in Fig. 6, cannot be reached using VEM. This is supported by the fit of the data using

$$
\left(1 / k-1 / k_{0}\right) \propto \exp \left(\beta N_{V}\right),
$$

yielding a minimum $\mathrm{DOH}$ of $1 / k_{0}=0.43$, which is larger than the $1 / k$-value of the homogeneous BD-microstructure. The second fit parameter and the corresponding correlation coefficient are $\beta=-0.25 \times 10^{-3}$ and $R^{2}=0.99$, respectively. The reason for this asymptotic behavior is most probably related to the VEM-algorithm itself in combination with the chosen targeted mean coordination number $C N=4.7$. As shown in [24], $C N$ as a function of the void-particle radius follows a characteristic step-like shape with $C N_{i}$ the coordination number at the curve's inflection point. $C N_{i}$ was found to decrease for increasing void-particle numbers $N_{V}$. Above the inflection point, $C N$ scales as a power law with exponent 0.37 , independently of $N_{V}$. Therefore it becomes increasingly difficult to reach a targeted $C N>C N_{i}$ toward larger values of $N_{V}$, at least without accepting considerable particle overlaps and consequently high internal stresses. The mean particle overlap, as determined by the maximum in the pair-correlation function was found to be $1.4 \%$ of the particle diameter $d_{S}$ in the case of $N_{V}=13,000$ and $C N=4.7$. For $N_{V}=16,000$ the mean overlap is $1.5 \% d_{S}$, however with a slightly lower $C N$ of 4.63 . The maximum particle overlap is below $2 \% d_{S}$ for all microstructure with $N_{V} \leq$ 8,000 and increases to roughly $4 \% d_{S}$ for $N_{V}=16,000$. For $N_{V}=20,000$ a mean and maximum overlap of 2 and $5 \% d_{S}$, respectively was already found for $C N=4.57$, which led to the conclusion that $N_{V}=16,000$ and thus a void- to structural particle number ration $n^{\max }=2.0$ constitutes the upper limit of VEM, for a volume fraction of $\Phi_{S}=0.4$.

The lower limit of $N_{V}$, at around 1,000 , is mainly imposed by the decreasing fit quality obtained in the case of the pore size and Voronoi volume distribution, as expressed in terms of the $R^{2}$-values (Table 2). The minimum void- to structural particle number ratio in the case of $\Phi_{S}=0.4$ is thus $n^{\text {min }}=0.125$.

\subsection{Influence of the volume fraction on the $\mathrm{DOH}$}

In the following, the DOH-range is investigated for volume fractions above 0.4. In particular, VEM-microstructures with volume fractions $\Phi_{S}=0.45,0.50,0.55$ and 0.60 have been generated and analyzed analogously to the structures with $\Phi_{S}=0.4$. This range of $\Phi_{S}$ was chosen since it is of particular interest for further simulations of the microstructure-dependent properties of coagulated colloidal suspensions [16]. As mentioned in Sect. 2.1, the targeted average coordination numbers for the structures with $\Phi_{S}>0.4$ are obtained using a linear interpolation between $C N_{0.4}=4.7$ and $C N_{R C P}=6.0$ yielding approximately 5.0, 5.25, 5.5 and 5.75 , respectively.

In this section, the DOH is measured by means of parameter $a$ obtained by fitting the cumulative pore size distribution using the complementary error function given in Eq. 2. This method was chosen because it yielded the best fit results over the whole range of volume fractions above $0.4\left(R^{2}>\right.$ 0.999 for all fits). The results are compiled in Table 3. For each volume fraction, an increasing void-particle number entails a decreasing parameter $a$ and therefore a decreasing DOH. This is summarized in Fig. 7 presenting the $\mathrm{DOH}$ values of these structures, as measured by parameter $a$ (circles). Particular interest is given to the maximum and minimum values of $a$ as a function of $\Phi_{S}: a^{\max }\left(\Phi_{S}\right)$ and $a^{\min }\left(\Phi_{S}\right)$, respectively. Our results suggest linear dependencies as illustrated by the solid lines in Fig. 7. The respective fits yield:

$$
\begin{aligned}
& a^{\max }\left(\Phi_{S}\right)=-2.16 \Phi_{S}+1.47 \\
& a^{\min }\left(\Phi_{S}\right)=-1.11 \Phi_{S}+0.842 .
\end{aligned}
$$


Table 3 DOH-measure $a$ as a function of the void-particle number $N_{V}$ for various volume fractions $\Phi_{S}>0.4$

\begin{tabular}{rllll}
\hline$N_{V}$ & $\Phi_{S}=0.45$ & $\Phi_{S}=0.50$ & $\Phi_{S}=0.55$ & $\Phi_{S}=0.60$ \\
\hline 500 & - & - & 0.283 & 0.191 \\
1,000 & 0.493 & 0.377 & 0.267 & - \\
2,000 & 0.441 & 0.345 & 0.256 & - \\
4,000 & 0.397 & 0.320 & 0.247 & - \\
6,000 & 0.376 & 0.307 & 0.242 & - \\
8,000 & 0.364 & 0.299 & 0.238 & - \\
10,000 & 0.356 & 0.295 & 0.236 & - \\
12,000 & 0.351 & 0.292 & 0.234 & - \\
16,000 & 0.340 & 0.285 & - & \\
\hline
\end{tabular}

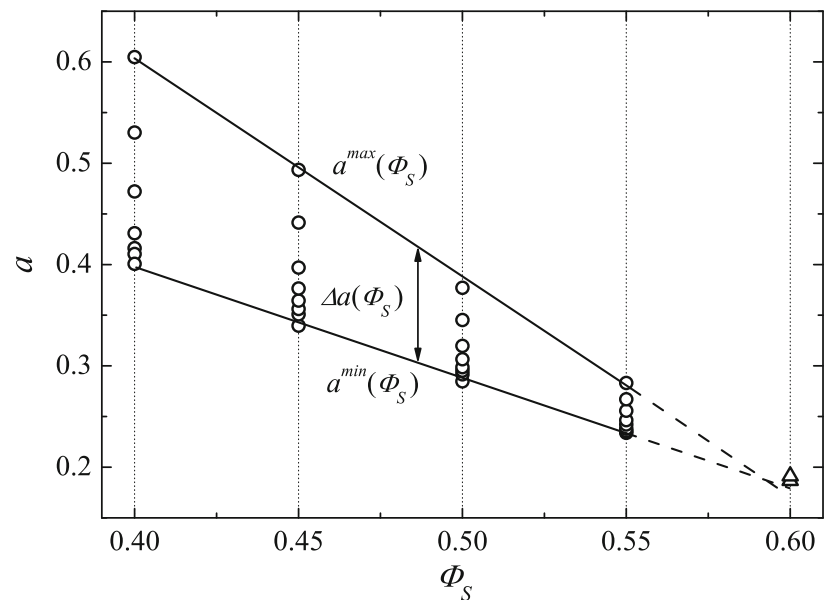

Fig. 7 DOH-range for volume fractions $\Phi_{S}$ between 0.4 and 0.6 as expressed by measure $a$ obtained from the pore size distribution

which, for the DOH-range measured by parameter $a$, results in

$\Delta a\left(\Phi_{S}\right)=-1.05 \Phi_{S}+0.624$

Equation 7 describes the narrowing of the DOH-range toward increasing volume fraction. With respect to the DOH-range at $\Phi_{S}=0.4$, the ranges for $\Phi_{S}=0.45,0.5$ and 0.55 are reduced by 30,55 and $76 \%$, respectively. The linear extrapolations of $a^{\max }\left(\Phi_{S}\right)$ and $a^{\min }\left(\Phi_{S}\right)$ toward higher volume fractions are shown as dashed lines in Fig. 7. The lines intersect at a volume fraction of 0.594 with corresponding $\mathrm{DOH}-$ value $a_{0}=0.183$.

The DOH-range for a random packing of monosized spheres is thus reduced to a unique value at this volume fraction. This was confirmed using two additional VEM-microstructure at $\Phi_{S}=0.6$ and using 500 and 8,000 void particles (triangles in Fig. 7). The DOH-measure $a$ for these two structures yields 0.187 and 0.191 , respectively. The range is thus narrowed down to roughly $2 \%$ with respect to the $\mathrm{DOH}$-range at $\Phi_{S}=0.4$.
The decreasing DOH-range toward larger volume fractions is qualitatively explained by the decrease of the pore space volume required for particle rearrangements. The volume fraction of 0.6, however, is below the RCP-limit, which was assumed to be the volume fraction, at which variations in DOH become zero. In [37], for example, the nature of the RCP limit was studied by means of a statistical mechanics approach and it was found that the entropy and thus the number of accessible configurations of a disordered packing of monosized spheres reaches a minimum at the RCP limit. Additionally, a sharp decrease in parameter $k$ was found as the volume fraction crosses the RLP limit in [38]. Following our interpretation of $k$, this sharp decrease corresponds to a sharp increase in DOH, which is not found in our data. More detailed studies are required in order to investigate these differences.

\section{Summary and conclusions}

In this paper, the $\mathrm{DOH}$ of microstructures generated using VEM has been analyzed and quantified using three distinct techniques based on the pore size distribution, the densityfluctuation method and the Voronoi volume distribution. In particular, the influence of the void-particle number and the volume fraction on the $\mathrm{DOH}$ was investigated.

The various pore size distributions were fitted using a complementary error function achieving very good fit results over the whole range of volume fractions investigated in this study. For all volume fractions, parameter $a$, corresponding to the width of the pore size distribution, increases for decreasing $N_{V}$, thereby reflecting the transition toward a higher DOH. For this range of volume fractions, the VEM thus allows for a generation of particle arrangements, for which the $\mathrm{DOH}$ can be controlled quasi-continuously over a broad range by means of the void-particle number. In particular, the $\mathrm{DOH}$ range becomes narrower and shifts to lower values as a function of increasing volume fraction. Our results indicate that 
the DOH-range is reduced to a single value at a volume fraction of roughly 0.6 , which, interestingly, is below the RCP limit. Further studies are required in order to analyze the behavior of the DOH and its range for volume fractions toward and beyond the RCP limit.

For VEM-structures with volume fraction 0.4 , the significant influence of the void-particle number on the DOH as measured by parameter $a$ is confirmed by the densityfluctuation method and the Voronoi volume distribution. $I_{d f}$, given by the integral over the various density fluctuation curves, increases for decreasing $N_{V}$. Parameter $k$, characterizing the shape of the Voronoi volume distributions, decreases for decreasing $N_{V}$.

The DOH of the VEM-microstructures with a volume fraction of 0.4 has been compared to that of BD-microstructures [26,27] analyzed in [25]. The structures generated using BD-simulations represent coagulated colloidal particle structures where the coagulation was simulated using widely accepted physical laws and theories. The DOH of the BDmicrostructures was shown to be closely related to the presence and depth of a secondary minimum in the DLVO potential, which is determined by the particles' surface potential. In contrast to BD, VEM is a stochastic method that mimics the swelling of ASP particles in experiment, having the advantage of its computational effectiveness. The DOHrange covered by VEM in terms of parameter $a$, characterizing the width of the pore size distribution, is approximately $20 \%$ broader and shifted to slightly higher values than the DOH-range of these BD-microstructures. The most homogeneous BD-microstructure, for which a surface potential $\Psi_{0}=0 \mathrm{mV}$ was used, could not be reproduced by VEM in terms of heterogeneity. A further decrease of the $\mathrm{DOH}$ through an increase in $N_{V}$ is mostly impeded by the choice of the targeted coordination number (roughly 4.7). For void-particle numbers exceeding 16,000 , this value cannot be attained without considerable particle overlap and therefore internal stress in the microstructure. On the other hand, VEM allows generating more heterogeneous microstructures than these BD-simulations, where the increasing depth of the secondary minimum toward higher surface potentials inhibits a complete coagulation.

This study has undoubtedly shown, that the void-particle number constitutes an important simulation parameter of VEM with regard to the DOH of the final microstructure. This parameter indeed allows for a quasi-continuous control of the DOH over a broad range of volume fractions. In order to compare the structural arrangement and to assess the influence of the void-particle number on the final microstructures, the concept of the DOH itself has proven particularly useful. The comparison of VEM- and BD-generated structures at a volume fraction of 0.4 allows concluding that VEM nicely reproduces the microstructures of coagulated colloids. Thereby, VEM facilitates the further study of the microstruc- ture-dependent mechanical properties of coagulated colloidal structures or granular matter in general.

\section{References}

1. Yun, T.S., Santamarina, J.C., Ruppel, C.: Mechanical properties of sand, silt and clay containing tetrahydrofuran hydrate. J. Geophys. Res. 112, B04106 (2007)

2. Touiti, L., Bouassida, M., Van Impe, W.: Discussion on Tunis soft soil sensitivity. Geotech. Geol. Eng. 27(5), 631-643 (2009)

3. Mezzenga, R., Schurtenberger, P., Burbidge, A., Michel, M.: Understanding foods as soft materials. Nat. Mater. 4, 729740 (2005)

4. Barbesta, F., Bousfield, D.W., Rigdahl, M.: Modeling of rheological properties of coating colors. J. Rheol. 45(1), 139-160 (2001)

5. Lee, Y.S., Wagner, N.J.: Dynamic properties of shear thickening colloidal suspensions. Rheol. Acta 42(3), 199-208 (2003)

6. Barnes, H.A.: Thixotropy-a review. J. Non-Newtonian Fluid Mech. 70, 1-33 (1997)

7. Abou, B., Bonn, D., Meunier, J.: Aging dynamics in a colloidal glass. Phys. Rev. E 64(2), 021510 (2001)

8. Zaccone, A., Lattuada, M., Wu, H., Morbidelli, M.: Theoretical elastic moduli for disordered packings of interconnected spheres. J. Chem. Phys. 127(17), 174512 (2007)

9. Gardiner, B.S., Tordesillas, A.: Effect of particle size distribution in a three-dimensional micropolar continuum model of granular media. Powder Technol. 161(2), 110-121 (2006)

10. Silbert, L.E., Ertaş, D., Grest, G.S., Halsey, T.C., Levine, D.: Geometry of frictionless and frictional sphere packings. Phys. Rev. E 65(3), 031304 (2002)

11. Martin, C.L., Bordia, R.K.: Influence of adhesion and friction on the geometry of packings of spherical particles. Phys. Rev. E 77(3), 031307 (2008)

12. Atman, A.P.F., Brunet, P., Geng, J., Reydellet, G., Combe, G., Claudin, P., Behringer, R.P., Clément, E.: Sensitivity of the stress response function to packing preparation. J. Phys. Condens. Matter 17, S2391-S2403 (2005)

13. Franks, G.V., Zhou, Y., Yan, Y., Jameson, G., Biggs, S.: Effect of aggregate size on sediment bed rheological properties. Phys. Chem. Chem. Phys. 6(18), 4490-4498 (2004)

14. Wyss, H.M., Tervoort, E.V., Gauckler, L.J.: Mechanics and microstructures of concentrated particle gels. J. Am. Ceram. Soc. 88(9), 2337-2348 (2005)

15. Wyss, H.M., Tervoort, E., Meier, L.P., Müller, M., Gauckler, L.J.: Relation between microstructure and mechanical behavior of concentrated silica gels. J. Colloid Interface Sci. 273(2), 455$462(2004)$

16. Wyss, H.M., Deliormanli, A.M., Tervoort, E., Gauckler, L.J.: Influence of microstructure on the rheological behavior of dense particle gels. AIChE J. 51(1), 134-141 (2005)

17. Gauckler, L.J., Graule, Th., Baader, F.: Ceramic forming using enzyme catalyzed reactions. Mater. Chem. Phys. 61(1), 78-102 (1999)

18. Tervoort, E., Tervoort, T.A., Gauckler, L.J.: Chemical aspects of direct coagulation casting of alumina suspensions. J. Am. Ceram. Soc. 87(8), 1530-1535 (2004)

19. Hesselbarth, D., Tervoort, E., Urban, C., Gauckler, L.J.: Mechanical properties of coagulated wet particle networks with alkali-swellable thickeners. J. Am. Ceram. Soc. 84(8), 1689-1695 (2001)

20. Agnolin, I., Roux, J.-N.: Internal states of model isotropic granular packings. I. Assembling process, geometry and contact networks. Phys. Rev. E 76(1), 061302 (2007) 
21. Jerkins, M., Schröter, M., Swinney, H.L., Senden, T.J., Saadatfar, M., Aste, T.: Onset of mechanical stability in random packings of frictional spheres. Phys. Rev. Lett. 101(1), 018301 (2008)

22. Bagi, K.: An algorithm to generate random dense arrangements for discrete element simulations of granular assemblies. Granul. Matter 7(1), 31-43 (2005)

23. Luding, S.: Contact models for very loose granular materials. In: Eberhard, P. (ed.) Symposium on Multiscale Problems in Multibody System Contacts, pp. 135-150. Springer, Heidelberg, ISBN 978-1-4020-5980-3 (2007)

24. Schenker, I., Filser, F.T., Herrmann, H.J., Gauckler, L.J.: Generation of porous particle structures using the void expansion method. Granul. Matter 11(3), 201-208 (2009)

25. Schenker, I., Filser, F.T., Aste, T., Herrmann, H.J., Gauckler, L.J.: Quantification of the heterogeneity of particle packings. Phys. Rev. E 80(2), 021302 (2009)

26. Hütter, M.: Local structure evolution in particle network formation studied by Brownian dynamics simulation. J. Colloid Interface Sci. 231(2), 337-350 (2000)

27. Hütter, M.: Brownian dynamics simulation of stable and of coagulating colloids in aqueous suspension, Ph.D. thesis no. 13107, ETH Zurich, Switzerland (1999)

28. Russel, W.B., Saville, D.A., Schowalter, W.R.: Colloidal dispersions, Cambridge University Press, New York (March 1989)

29. PFC $^{3 \mathrm{D}}$ User's Manual, Itasca Consulting Group, Inc., Minneapolis, Minnesota, USA (1995)
30. Cundall, P.A., Strack, O.D.L.: A discrete numerical model for granular assemblies. Géotechnique 29(1), 47-65 (1979)

31. Brown, E.T.: Analytical and Computational Methods in Engineering Rock Mechanics, Ed. Allen \& Unwin, London (1987)

32. Song, C., Wang, P., Makse, H.A.: A phase diagram for jammed matter. Nature 453(29), 629-632 (2008)

33. Torquato, S., Lu, B., Rubinstein, J.: Nearest-neighbor distribution functions in many-body systems. Phys. Rev. A 41(4), 20592075 (1990)

34. Voronoi, G.: Recherches sur les paralléloèdres primitives. J. Reine Angew. Math. 134, 198-287 (1908)

35. Barber, C.B., Dobkin, D.P., Huhdanpaa, H.: The quickhull algorithm for convex hulls. ACM Trans. Math. Softw. 22(4), 469483 (1996)

36. Aste, T., Di Matteo, T.: Emergence of Gamma distributions in granular materials and packing models. Phys. Rev. E 77(2), 021309 (2008)

37. Anikeenko, A.V., Medvedev, N.N., Aste, T.: Structural and entropic insights into the nature of the random-close-packing limit. Phys. Rev. E 77(3), 031101 (2008)

38. Aste, T., Di Matteo, T.: Structural transitions in granular packs: statistical mechanics and statistical geometry investigations. Eur. Phys. J. B 64, 511-517 (2008) 